Pacific

Journal of

Mathematics

REAL PALEY-WIENER THEOREMS FOR THE INVERSE FOURIER TRANSFORM ON A RIEMANNIAN

SYMMETRIC SPACE

Nils Byrial ANDERsen

Volume 213 No. 1

January 2004 


\title{
REAL PALEY-WIENER THEOREMS FOR THE INVERSE FOURIER TRANSFORM ON A RIEMANNIAN SYMMETRIC SPACE
}

\author{
NiLs Byrial ANDERSEN
}

\begin{abstract}
We prove real Paley-Wiener theorems for the inverse Fourier transform on a semisimple Riemannian symmetric space $G / K$ of the noncompact type. The functions on $G / K$ whose Fourier transform has compact support are characterised by a $L^{2}$ growth condition. We also obtain real Paley-Wiener theorems for the inverse spherical transform.
\end{abstract}

\section{Introduction.}

The classical Fourier transform $\mathcal{F}_{\mathrm{cl}}$ is an isomorphism of the Schwartz space $\mathcal{S}\left(\mathbb{R}^{k}\right)$ onto itself. The space $C_{c}^{\infty}\left(\mathbb{R}^{k}\right)$ of smooth functions with compact support is dense in $\mathcal{S}\left(\mathbb{R}^{k}\right)$, and the classical Paley-Wiener theorem characterises the image of $C_{c}^{\infty}\left(\mathbb{R}^{k}\right)$ under $\mathcal{F}_{\mathrm{cl}}$ as rapidly decreasing functions having an holomorphic extension to $\mathbb{C}^{k}$ of exponential type. Since $\mathbb{R}^{k}$ is self-dual, the same theorem also applies to the inverse Fourier transform.

Let $G$ be a noncompact semisimple Lie group and $K$ a maximal compact subgroup of $G$. The Fourier transform $\mathcal{F}$ on the Riemannian symmetric space $X=G / K$ is an analogue of the classical Fourier transform on $\mathbb{R}^{k}$. A Paley-Wiener theorem for the Fourier transform $\mathcal{F}$, which characterises the image of $C_{c}^{\infty}(X)$ under $\mathcal{F}$ in terms of holomorphic extensions and growth behaviour, as in the classical case, was proved by Helgason, see [7]. Furthermore, the $L^{2}$-Schwartz space $\mathcal{S}^{2}(X)$ contains $C_{c}^{\infty}(X)$ as a dense subspace and $\mathcal{F}$ is an isomorphism of $\mathcal{S}^{2}(X)$ onto some generalised Schwartz space, see $[4]$.

Unlike the classical case, however, we can not use a duality argument to deduce a Paley-Wiener theorem for the inverse Fourier transform. So how can we characterise the functions whose Fourier transform $\mathcal{F}$ has compact support?

The Fourier transform on $X$ reduces to the spherical transform $\mathcal{H}$ on $G$ when restricted to $K$-invariant functions. The paper [8] provides an answer to the above question for the spherical transform on Schwartz functions in the rank one and complex cases. The characterisation is in analogy with the classical Paley-Wiener theorem given in terms of meromorphic extensions and growth conditions. 
In this paper we prove (real) Paley-Wiener theorems for the inverse Fourier transform for general Riemannian symmetric spaces, i.e., we characterise, as a subset of $L^{2}(X)$, the set of functions $f$ on $X$ whose Fourier transform $\mathcal{F} f$ has compact support. More precisely, $f \in C^{\infty}(X)$ has to satisfy

$$
\lim _{n \rightarrow \infty}\left\|\Delta^{n} f\right\|_{2}^{1 / 2 n}<\infty
$$

where $\Delta$ is the Laplace-Beltrami operator (and $(1+|\cdot|)^{n} f \in L^{2}(X)$ for all $n \in \mathbb{N} \cup\{0\}$ if we also want the Fourier image to be smooth). Specialising to bi- $K$-invariant functions yields (real) Paley-Wiener theorems for the inverse spherical transform for general noncompact semisimple Lie groups.

Our approach is based on real analysis techniques developed by $\mathrm{H}$. H. Bang, see [2] and [3], and V.K. Tuan, see [10]. Also see [11] for a history and overview of (real) Paley-Wiener theorems for certain transforms (Fourier, Mellin, Hankel...) on $\mathbb{R}$. In particular we use Parseval's formula, intertwining properties of $\mathcal{F}$, and the following characterisation of the radius of the support of a function $g$ on $\mathbb{R}^{n}$ :

$$
\sup _{\lambda \in \operatorname{supp} g}\|\lambda\|=\lim _{n \rightarrow \infty}\left\{\int_{\mathbb{R}^{n}}\|\lambda\|^{4 n}|g(\lambda)|^{2} d \lambda\right\}^{1 / 4 n} .
$$

For completeness and comparison, we first consider the Fourier transform on $\mathbb{R}^{k}$. The results here are originally due to H.H. Bang, see [2] and [3], and V.K. Tuan, see [10]. Notice the beautiful symmetry between the (statements of the) results for the various transforms.

\section{The Fourier transform on $\mathbb{R}^{k}$.}

For background and details, please see $[\mathbf{9}$, Chapter 7$]$. Let $\mathcal{F}_{\mathrm{cl}}$ denote the classical Fourier transform on $\mathbb{R}^{k}$ :

$$
\mathcal{F}_{\mathrm{cl}} f(\lambda):=\int_{\mathbb{R}^{k}} f(x) e^{-i \lambda \cdot x} d x
$$

defined for nice functions $f$, for all $\lambda \in \mathbb{C}^{k}$ for which the above integral makes sense. Let $\Delta=\frac{d^{2}}{d x_{1}^{2}}+\cdots+\frac{d^{2}}{d x_{k}^{2}}$ denote the Laplacian on $\mathbb{R}^{k}$ and let $\mathcal{S}\left(\mathbb{R}^{k}\right)$ denote the Schwartz space of rapidly decreasing differentiable functions. Then $\mathcal{F}_{\mathrm{cl}}(\Delta f)(\lambda)=-\|\lambda\|^{2} \mathcal{F}_{\mathrm{cl}} f(\lambda),\left(\lambda \in \mathbb{R}^{k}\right)$, for all $f \in \mathcal{S}\left(\mathbb{R}^{k}\right)$, and the Fourier transform is an isomorphism of $\mathcal{S}\left(\mathbb{R}^{k}\right)$ onto itself, with inverse given by:

$$
\mathcal{F}_{\mathrm{cl}}^{-1} g(x)=(2 \pi)^{-k} \int_{\mathbb{R}^{k}} g(\lambda) e^{i \lambda \cdot x} d \lambda, \quad\left(x \in \mathbb{R}^{k}\right)
$$


for $g \in \mathcal{S}\left(\mathbb{R}^{k}\right)$. Parseval's formula states that

$$
\begin{aligned}
\left\langle f_{1}, f_{2}\right\rangle & :=\int_{\mathbb{R}^{k}} f_{1}(x) \overline{f_{2}(x)} d x=(2 \pi)^{-k} \int_{\mathbb{R}^{k}} \mathcal{F}_{\mathrm{cl}} f_{1}(\lambda) \overline{\mathcal{F}_{\mathrm{cl}} f_{2}(\lambda)} d \lambda \\
& =:\left\langle\mathcal{F}_{\mathrm{cl}} f_{1}, \mathcal{F}_{\mathrm{cl}} f_{2}\right\rangle,
\end{aligned}
$$

for $f_{1}, f_{2} \in \mathcal{S}\left(\mathbb{R}^{k}\right)$, which implies that $\|f\|_{2}=\left\|\mathcal{F}_{\text {cl }} f\right\|_{2}$, for all $f \in \mathcal{S}\left(\mathbb{R}^{k}\right)$, and hence that the Fourier transform extends to an isometry from $L^{2}\left(\mathbb{R}^{k}\right)$ onto itself.

Let $f \in C^{\infty}\left(\mathbb{R}^{k}\right)$ such that $\Delta^{n} f \in L^{2}\left(\mathbb{R}^{k}\right)$ for all $n \in \mathbb{N} \cup\{0\}$ and let $f_{2} \in C_{c}^{\infty}\left(\mathbb{R}^{k}\right)$. Then:

$$
\begin{aligned}
\left\langle\mathcal{F}_{\mathrm{cl}}(\Delta f), \mathcal{F}_{\mathrm{cl}} f_{2}\right\rangle & =\left\langle\Delta f, f_{2}\right\rangle=\left\langle f, \Delta f_{2}\right\rangle=\left\langle\mathcal{F}_{\mathrm{cl}} f, \mathcal{F}_{\mathrm{cl}}\left(\Delta f_{2}\right)\right\rangle \\
& =\left\langle\mathcal{F}_{\mathrm{cl}} f,-\|\lambda\|^{2} \mathcal{F}_{\mathrm{cl}} f_{2}\right\rangle=\left\langle-\|\lambda\|^{2} \mathcal{F}_{\mathrm{cl}} f, \mathcal{F}_{\mathrm{cl}} f_{2}\right\rangle,
\end{aligned}
$$

and we conclude that $\mathcal{F}_{\mathrm{cl}}(\Delta f)(\lambda)=-\|\lambda\|^{2} \mathcal{F}_{\mathrm{cl}} f(\lambda)$ a.e., by a density argument, whence $\mathcal{F}_{\mathrm{cl}}\left(\Delta^{n} f\right)(\lambda)=(-1)^{n}\|\lambda\|^{2 n} \mathcal{F}_{\mathrm{cl}} f(\lambda)$ a.e., and

$$
\int_{\mathbb{R}^{k}}\left|\Delta^{n} f(x)\right|^{2} d x=(2 \pi)^{-k} \int_{\mathbb{R}^{k}}\|\lambda\|^{4 n}\left|\mathcal{F}_{\mathrm{cl}} f(\lambda)\right|^{2} d \lambda,
$$

for all $\in \mathbb{N} \cup\{0\}$.

We define the support, $\operatorname{supp} g$, of $g \in L^{2}\left(\mathbb{R}^{k}\right)$ to be the smallest closed set, outside which the function $g$ vanishes almost everywhere, and $R_{g}:=$ $\sup _{\lambda \in \operatorname{supp} g}\|\lambda\|$ to be the radius of the support of $g ; R_{g}$ is finite if, and only if, $g$ has compact support.

Lemma 2.1. Let $g \in L^{2}\left(\mathbb{R}^{k}\right)$ such that $\|\lambda\|^{2 n} g(\lambda) \in L^{2}\left(\mathbb{R}^{k}\right)$ for all $n \in$ $\mathbb{N} \cup\{0\}$. Then

$$
R_{g}=\lim _{n \rightarrow \infty}\left\{\int_{\mathbb{R}^{k}}\|\lambda\|^{4 n}|g(\lambda)|^{2} d \lambda\right\}^{1 / 4 n} .
$$

Proof. Assume $g$ has compact support with $R_{g}>0$. Then:

$$
\begin{aligned}
& \limsup _{n \rightarrow \infty}\left\{\int_{\mathbb{R}^{k}}\|\lambda\|^{4 n}|g(\lambda)|^{2} d \lambda\right\}^{1 / 4 n} \\
& \leq R_{g} \limsup _{n \rightarrow \infty}\left\{\int_{\|\lambda\| \leq R_{g}}|g(\lambda)|^{2} d \lambda\right\}^{1 / 4 n}=R_{g} .
\end{aligned}
$$

On the other hand,

$$
\int_{R_{g}-\varepsilon \leq\|\lambda\| \leq R_{g}}|g(\lambda)|^{2} d \lambda>0
$$


for any $\varepsilon>0$, hence

$$
\begin{aligned}
& \liminf _{n \rightarrow \infty}\left\{\int_{\mathbb{R}^{k}}\|\lambda\|^{4 n}|g(\lambda)|^{2} d \lambda\right\}^{1 / 4 n} \\
& \geq \liminf _{n \rightarrow \infty}\left\{\int_{R_{g}-\varepsilon \leq\|\lambda\| \leq R_{g}}\|\lambda\|^{4 n}|g(\lambda)|^{2} d \lambda\right\}^{1 / 4 n} \\
& \geq\left(R_{g}-\varepsilon\right) \liminf _{n \rightarrow \infty}\left\{\int_{R_{g}-\varepsilon \leq\|\lambda\| \leq R_{g}}|g(\lambda)|^{2} d \lambda\right\}^{1 / 4 n}=R_{g}-\varepsilon
\end{aligned}
$$

and thus

$$
\lim _{n \rightarrow \infty}\left\{\int_{\mathbb{R}_{n}}\|\lambda\|^{4 n}|g(\lambda)|^{2} d \lambda\right\}^{1 / 4 n}=R_{g} .
$$

Now assume that $g$ has unbounded support. Then

$$
\int_{\|\lambda\| \geq N}|g(\lambda)|^{2} d \lambda>0
$$

for any $N>0$, so:

$$
\begin{aligned}
& \liminf _{n \rightarrow \infty}\left\{\int_{\mathbb{R}^{k}}\|\lambda\|^{4 n}|g(\lambda)|^{2} d \lambda\right\}^{1 / 4 n} \\
& \geq \liminf _{n \rightarrow \infty}\left\{\int_{\|\lambda\| \geq N}\|\lambda\|^{4 n}|g(\lambda)|^{2} d \lambda\right\}^{1 / 4 n} \\
& \geq N \liminf _{n \rightarrow \infty}\left\{\int_{\|\lambda\| \geq N}|g(\lambda)|^{2} d \lambda\right\}^{1 / 4 n}=N
\end{aligned}
$$

for arbitrary $N>0$, and we conclude that

$$
\liminf _{n \rightarrow \infty}\left\{\int_{\mathbb{R}^{k}}\|\lambda\|^{4 n}|g(\lambda)|^{2} d \lambda\right\}^{1 / 4 n}=\infty .
$$

Let $L_{c}^{2}\left(\mathbb{R}^{k}\right)$ denote the subspace of $L^{2}\left(\mathbb{R}^{k}\right)$ of functions with compact support and let $L_{R}^{2}\left(\mathbb{R}^{k}\right):=\left\{g \in L_{c}^{2}\left(\mathbb{R}^{k}\right) \mid R_{g}=R\right\}$. Let also $C_{R}^{\infty}\left(\mathbb{R}^{k}\right):=$ $\left\{g \in C_{c}^{\infty}\left(\mathbb{R}^{k}\right) \mid R_{g}=R\right\}$.

Definition 2.2. We define the $L^{2}$-Paley-Wiener space $\mathrm{PW}^{2}\left(\mathbb{R}^{k}\right)$ to be the space of all functions $f \in C^{\infty}\left(\mathbb{R}^{k}\right)$ satisfying:
(a) $\Delta^{n} f \in L^{2}\left(\mathbb{R}^{k}\right)$ for all $n \in \mathbb{N} \cup\{0\}$.
(b) $R_{f}^{\Delta}:=\lim _{n \rightarrow \infty}\left\|\Delta^{n} f\right\|_{2}^{1 / 2 n}<\infty$. 
Let also $\mathrm{PW}_{R}^{2}\left(\mathbb{R}^{k}\right):=\left\{f \in \mathrm{PW}^{2}\left(\mathbb{R}^{k}\right) \mid R_{f}^{\Delta}=R\right\}$, for $R \geq 0$.

The proof of Theorem 2.3 below shows that the limit in (b) above is welldefined. The real version of the $L^{2}$-Paley-Wiener theorem for the inverse Fourier transform can now be formulated as follows:

Theorem 2.3. The inverse Fourier transform $\mathcal{F}_{\mathrm{cl}}^{-1}$ is a bijection of $L_{c}^{2}\left(\mathbb{R}^{k}\right)$ onto $\mathrm{PW}^{2}\left(\mathbb{R}^{k}\right)$, mapping $L_{R}^{2}\left(\mathbb{R}^{k}\right)$ onto $\mathrm{PW}_{R}^{2}\left(\mathbb{R}^{k}\right)$.

Proof. Let $g \in L_{R}^{2}\left(\mathbb{R}^{k}\right)$. Then $\|\lambda\|^{n} g(\lambda) \in L^{1}\left(\mathbb{R}^{k}\right)$ for all $n \in \mathbb{N} \cup\{0\}$, and $\mathcal{F}_{\text {cl }}^{-1} g \in C_{o}^{\infty}\left(\mathbb{R}^{k}\right)$. We also have $\Delta^{n}\left(\mathcal{F}_{\mathrm{cl}}^{-1} g\right)=\mathcal{F}_{\mathrm{cl}}^{-1}\left((-1)^{n}\|\lambda\|^{2 n} g\right) \in L^{2}\left(\mathbb{R}^{k}\right)$ for all $n \in \mathbb{N} \cup\{0\}$, by the formula for $\mathcal{F}_{\mathrm{cl}}^{-1}$, and (1) thus yields:

$$
\begin{aligned}
& \lim _{n \rightarrow \infty}\left\{\int_{\mathbb{R}^{k}}\left|\Delta^{n}\left(\mathcal{F}_{\mathrm{cl}}^{-1} g\right)(x)\right|^{2} d x\right\}^{1 / 4 n} \\
& =\lim _{n \rightarrow \infty}\left\{(2 \pi)^{-k} \int_{\mathbb{R}^{k}}\|\lambda\|^{4 n}|g(\lambda)|^{2} d \lambda\right\}^{1 / 4 n}=R,
\end{aligned}
$$

whence $\mathcal{F}_{\mathrm{cl}}^{-1} g \in \mathrm{PW}_{R}^{2}\left(\mathbb{R}^{k}\right)$.

Let now $f \in \mathrm{PW}_{R}^{2}\left(\mathbb{R}^{k}\right)$. Then $\mathcal{F}_{\mathrm{cl}}\left(\Delta^{n} f\right)(\lambda)=(-1)^{n}\|\lambda\|^{2 n} \mathcal{F}_{\mathrm{cl}} f(\lambda) \in$ $L^{2}\left(\mathbb{R}^{k}\right)$ for all $n \in \mathbb{N}$, and another application of (1) shows that

$$
\begin{aligned}
& \lim _{n \rightarrow \infty}\left\{(2 \pi)^{-k} \int_{\mathbb{R}^{k}}\|\lambda\|^{4 n}\left|\mathcal{F}_{\mathrm{cl}} f(\lambda)\right|^{2} d \lambda\right\}^{1 / 4 n} \\
& =\lim _{n \rightarrow \infty}\left\{\int_{\mathbb{R}^{k}}\left|\Delta^{n} f(x)\right|^{2} d x\right\}^{1 / 4 n}=R,
\end{aligned}
$$

and we conclude that $\mathcal{F}_{\text {cl }} f$ has compact support with $R_{\mathcal{F}_{\text {cl }} f}=R$.

Remark 2.4. The classical (complex) $L^{2}$-Paley-Wiener theorem implies that $\mathrm{PW}_{R}^{2}\left(\mathbb{R}^{k}\right)$ exactly consists of those $L^{2}\left(\mathbb{R}^{k}\right)$ functions that can be extended to holomorphic functions of exponential type $R$ on $\mathbb{C}^{k}$.

Remark 2.5. Let $f \in \mathrm{PW}^{2}(\mathbb{R})$. Then $\frac{d^{n}}{d x^{n}} f \in L^{p}(\mathbb{R})$ for all $n \in \mathbb{N} \cup\{0\}$, and:

$$
\lim _{n \rightarrow \infty}\left\|\frac{d^{n}}{d x^{n}} f\right\|_{p}^{1 / n}=R_{\mathcal{F}_{\mathrm{cl}} f}=R_{f}^{\Delta},
$$

for all $1 \leq p \leq \infty$. This follows from [2, Theorem 1]. Similar results hold for $\mathbb{R}^{k}, k>1$, see [3, Theorem 3] and [10, Theorem 4].

Definition 2.6. We define the Paley-Wiener space $\mathrm{PW}\left(\mathbb{R}^{k}\right)$ as the space of all functions $f \in C^{\infty}\left(\mathbb{R}^{k}\right)$ satisfying:

(a) $(1+|x|)^{m} \Delta^{n} f \in L^{2}\left(\mathbb{R}^{k}\right)$ for all $m, n \in \mathbb{N} \cup\{0\}$.

(b) $R_{f}^{\Delta}:=\lim _{n \rightarrow \infty}\left\|\Delta^{n} f\right\|_{2}^{1 / 2 n}<\infty$.

Let again $\mathrm{PW}_{R}\left(\mathbb{R}^{k}\right):=\left\{f \in \mathrm{PW}\left(\mathbb{R}^{k}\right) \mid R_{f}^{\Delta}=R\right\}$, for $R \geq 0$. 
We notice that the only difference between $\mathrm{PW}^{2}\left(\mathbb{R}^{k}\right)$ and $\mathrm{PW}\left(\mathbb{R}^{k}\right)$ is an extra requirement of polynomial decay, to help ensure that $\mathcal{F}_{\mathrm{cl}} f \in C^{\infty}\left(\mathbb{R}^{k}\right)$.

The real version of the Paley-Wiener theorem for the inverse Fourier transform is the following:

Theorem 2.7. The inverse Fourier transform $\mathcal{F}_{\mathrm{cl}}^{-1}$ is a bijection of $C_{c}^{\infty}\left(\mathbb{R}^{k}\right)$ onto $\mathrm{PW}\left(\mathbb{R}^{k}\right)$, mapping $C_{R}^{\infty}\left(\mathbb{R}^{k}\right)$ onto $\mathrm{PW}_{R}\left(\mathbb{R}^{k}\right)$.

Proof. Let $g \in C_{R}^{\infty}\left(\mathbb{R}^{k}\right)$, then $\mathcal{F}_{\text {cl }}^{-1} g \in \mathcal{S}\left(\mathbb{R}^{k}\right)$, and $\mathcal{F}_{\text {cl }}^{-1} g \in \mathrm{PW}_{R}^{2}\left(\mathbb{R}^{k}\right)$ by Theorem 2.3.

Let $f \in \mathrm{PW}_{R}\left(\mathbb{R}^{k}\right) \subset \mathrm{PW}_{R}^{2}\left(\mathbb{R}^{k}\right)$. Then $\mathcal{F}_{\text {cl }} f \in C^{\infty}\left(\mathbb{R}^{k}\right)$ since $f$ has polynomial decay, and $\mathcal{F}_{\text {cl }} f$ has compact support with $R_{\mathcal{F}_{\text {cl }} f}=R$ by Theorem 2.3.

\section{Lie group notation.}

In this section we introduce the Lie group notation we need in the next sections. We refer to [5], [6] and [7] for further details.

Let $G$ be a real connected noncompact semisimple Lie group with finite center and let $\theta$ be a Cartan involution of $G$. Then the fixed point group $K:=G^{\theta}$ is a maximal compact subgroup. Let $\mathfrak{g}$ and $\mathfrak{k}$ denote their Lie algebras, and let $\mathfrak{g}=\mathfrak{k} \oplus \mathfrak{p}$ be the Cartan decomposition of $\mathfrak{g}$ into the \pm 1 eigenspaces of $\theta$. The Killing form on $\mathfrak{g}$ induces an $\mathrm{Ad} K$-invariant scalar product on $\mathfrak{p}$ and hence a $G$-invariant Riemannian metric on $X:=G / K$. With this structure, $X$ becomes a Riemannian globally symmetric space of the noncompact type.

Fix a maximal abelian subspace $\mathfrak{a}$ of $\mathfrak{p}$. Denote its real dual by $\mathfrak{a}^{*}$ and its complex dual by $\mathfrak{a}_{\mathbb{C}}^{*}$. The Killing form of $\mathfrak{g}$ induces a scalar product $\langle\cdot, \cdot\rangle$ and hence a norm $\|\cdot\|$ on $\mathfrak{a}_{\mathbb{C}}$ and $\mathfrak{a}_{\mathbb{C}}^{*}$. Let $\Sigma \subset \mathfrak{a}^{*}$ denote the root system of $(\mathfrak{g}, \mathfrak{a})$ and let $W$ be the associated Weyl group. Choose a set $\Sigma_{+} \subset \Sigma$ of positive roots, let $\mathfrak{n}:=\bigoplus_{\alpha \in \Sigma_{+}} \mathfrak{g}_{\alpha}$ be the corresponding nilpotent subalgebra of $\mathfrak{g}$ and let $\mathfrak{a}_{+}:=\left\{H \in \mathfrak{a} \mid \alpha(H)>0 \forall \alpha \in \Sigma_{+}\right\}$be the positive Weyl chamber with $\overline{\mathfrak{a}_{+}}$it's closure. Denote by $\mathfrak{a}_{+}^{*}$ and $\overline{\mathfrak{a}_{+}^{*}}$ the similar cones in $\mathfrak{a}^{*}$, and define the element $\rho \in \mathfrak{a}^{*}$ by: $\rho(H):=\frac{1}{2} \sum_{\alpha \in \Sigma_{+}} m_{\alpha} \alpha(H), H \in \mathfrak{a}$, where $m_{\alpha}=\operatorname{dim} \mathfrak{g}_{\alpha}$.

Let $\mathfrak{g}=\mathfrak{k} \oplus \mathfrak{a} \oplus \mathfrak{n}$ be the Iwasawa decomposition of $\mathfrak{g}$ and $G=K A N=$ $N A K$ the corresponding Iwasawa decompositions of $\mathrm{G}$, where $A$ and $N$ are the Lie groups generated by $\mathfrak{a}$ and $\mathfrak{n}$ respectively. Every $g \in G$ can be represented as: $g=K \exp H(g) N=N \exp A(g) K$, where the projections onto the $A$-parts $A(g) \in \mathfrak{a}$ and $H(g) \in \mathfrak{a}$ are uniquely determined. We note that $A(g)=-H\left(g^{-1}\right)$. Let $M:=Z_{K}(\mathfrak{a})$, then $B:=K / M$ is a compact homogeneous space. We define the vector $A(x, b) \in \mathfrak{a}$ as $A(x, b):=A\left(k^{-1} g\right)$, for $x=g K \in X$ and $b=k M \in B$.

Put $A_{+}=\exp \left(\mathfrak{a}_{+}\right)$, then $\overline{A_{+}}=\exp \left(\overline{\mathfrak{a}_{+}}\right)$. The Cartan decomposition implies that the natural mapping from $K / M \times A_{+} \times K$ into $G=K \overline{A_{+}} K$ is a 
diffeomorphism onto its dense open image. We define the norm of an element $g \in G$ as: $|g|=\left|k_{1} \exp (H) k_{2}\right|=\|H\|$, with $H \in \overline{\mathfrak{a}_{+}}$; this is the $K$-invariant geodesic distance to the origin $e K$. We denote by $B_{R}:=\{g \in G|| g \mid \leq R\}$ the $K$-invariant ball of radius $R$ around $e$.

We identify functions on $X$ with right- $K$-invariant functions on $G$. We normalise the invariant measure on $X$ as:

$$
\int_{X} f(x) d x=\int_{K} \int_{\mathfrak{a}_{+}} \int_{K} f\left(k_{1} \exp (H) k_{2}\right) J(H) d k_{1} d H d k_{2},
$$

for $f \in C_{c}^{\infty}(X)$, where the Jacobian $J$ is given by: $J(H)=\prod_{\alpha \in \Sigma_{+}}\left(e^{\alpha(H)}-\right.$ $\left.e^{-\alpha(H)}\right)^{m_{\alpha}}, d H$ is the Lebesgue measure on $\mathfrak{a}$ and $d k$ is the measure on $K$ such that $\int_{K} d k=1$. We notice that $0 \leq J(H) \leq C e^{2 \rho(H)}$, for $H \in \overline{\mathfrak{a}_{+}}$, where $C$ is a positive constant.

The spherical functions $\varphi_{\lambda}, \lambda \in \mathfrak{a}_{\mathbb{C}}^{*}$, on $G$ are defined as:

$$
\varphi_{\lambda}(g):=\int_{K} e^{(i \lambda+\rho) A\left(k^{-1} g\right)} d k=\int_{K} e^{-(i \lambda+\rho) H\left(g^{-1} k\right)} d k .
$$

We note that $\varphi_{\lambda}$ is Weyl group invariant, $\varphi_{w \lambda}=\varphi_{\lambda}, w \in W$. Let $U(\mathfrak{g})$ denote the universal enveloping algebra of $\mathfrak{g}$. We write $D f(g)$ for the action of $D \in U(\mathfrak{g})$ on $f \in C^{\infty}(G)$ from the left at $g \in G$. The $L^{p}$-Schwartz space $\mathcal{S}^{p}(X), 0<p \leq 2$, is defined as the space of all functions $f \in C^{\infty}(X)$ such that:

$$
\sup _{g \in G}(1+|g|)^{m} \varphi_{o}(g)^{-\frac{2}{p}}|D f(g)|<\infty
$$

for all $D \in U(\mathfrak{g})$ and $m \in \mathbb{N} \cup\{0\}$. We can also characterise $\mathcal{S}^{p}(X)$ as the space of all functions $f \in C^{\infty}(X)$ satisfying:

$$
(1+|g|)^{m} D f(g) \in L^{p}(X),
$$

for all $D \in U(\mathfrak{g})$ and $m \in \mathbb{N} \cup\{0\}$. We note that $\mathcal{S}^{p}(X) \not \subset L^{q}(X)$ for $0<q<p \leq 2$.

\section{The Fourier transform.}

In this section, we recall some facts and theorems for the Fourier transform on a noncompact semisimple Riemannian symmetric space, see [7, Chapter 3] for details and references.

The Fourier transform of a function $f$ on $X$ is defined as:

$$
\mathcal{F} f(\lambda, b):=\int_{X} f(x) e^{(-i \lambda+\rho)(A(x, b))} d x,
$$

for all $\lambda \in \mathfrak{a}_{\mathbb{C}}^{*}, b \in B$ for which the integral exists. In particular, $\mathcal{F} f$ extends to a smooth function on $\mathfrak{a}_{\mathbb{C}}^{*} \times B$, holomorphic in the first variable, for $f \in$ $C_{c}^{\infty}(X)$, see also below. 
The plane wave eigenfunction

$$
e_{\lambda, b}(x):=e^{(i \lambda+\rho)(A(x, b))},
$$

is a joint eigenfunction of $\mathbb{D}(X)$, the commutative algebra of $G$-invariant differential operators on $X$, for all $\lambda \in \mathfrak{a}_{\mathbb{C}}^{*}, b \in B$, or, more precisely

$$
D e_{\lambda, b}=\Gamma(D)(i \lambda) e_{\lambda, b}, \quad \forall D \in \mathbb{D}(X), \quad\left(\lambda \in \mathfrak{a}_{\mathbb{C}}^{*}, b \in B\right)
$$

where $\Gamma: \mathbb{D}(X) \rightarrow S\left(\mathfrak{a}^{*}\right)^{W}$ is the Harish-Chandra isomorphism. In particular,

$$
\Delta e_{\lambda, b}=-\left(\langle\lambda, \lambda\rangle+\|\rho\|^{2}\right) e_{\lambda, b}, \quad\left(\lambda \in \mathfrak{a}_{\mathbb{C}}^{*}, b \in B\right)
$$

for the Laplace-Beltrami operator $\Delta$ on $X$, and hence

$$
\mathcal{F}(\Delta f)(\lambda, b)=-\left(\langle\lambda, \lambda\rangle+\|\rho\|^{2}\right) \mathcal{F} f(\lambda, b), \quad\left(\lambda \in \mathfrak{a}_{\mathbb{C}}^{*}, b \in B\right)
$$

for all $f \in C_{c}^{\infty}(X)$, by self-adjointness of $\Delta$, see also (6).

A $C^{\infty}$-function $\psi(\lambda, b)$ on $\mathfrak{a}_{\mathbb{C}}^{*} \times B$, holomorphic in $\lambda$, is called a holomorphic function of uniform exponential type $R$, if there exists a constant $R \geq 0$, such that, for each $N \in \mathbb{N}$, we have:

$$
\sup _{\lambda \in \mathfrak{a}_{\mathbb{C}}^{*}, b \in B} e^{-R|\Im \lambda|}(1+|\lambda|)^{N}|\psi(\lambda, b)|<\infty .
$$

The space of holomorphic functions of uniform exponential type $R$ will be denoted $\mathcal{H}_{R}\left(\mathfrak{a}_{\mathbb{C}}^{*} \times B\right)$ and we denote by $\mathcal{H}\left(\mathfrak{a}_{\mathbb{C}}^{*} \times B\right)$ their union over all $R>0$. Let furthermore $\mathcal{H}\left(\mathfrak{a}_{\mathbb{C}}^{*} \times B\right)^{W}$ denote the space of all functions $\psi \in \mathcal{H}\left(\mathfrak{a}_{\mathbb{C}}^{*} \times B\right)$ satisfying the symmetry condition:

$$
\int_{B} e^{(i w \lambda+\rho)(A(x, b))} \psi(w \lambda, b) d b=\int_{B} e^{(i \lambda+\rho)(A(x, b))} \psi(\lambda, b) d b,
$$

for $w \in W$ and all $\lambda \in \mathfrak{a}_{\mathbb{C}}^{*}, x \in X$.

The Paley-Wiener theorem states that the Fourier transform is a bijection of the space $C_{c}^{\infty}(X)$ onto the space $\mathcal{H}\left(\mathfrak{a}_{\mathbb{C}}^{*} \times B\right)^{W}$, with the following inversion formula:

$$
f(x)=\int_{\mathfrak{a}_{+}^{*}} \int_{B} e^{(i \lambda+\rho)(A(x, b))} \mathcal{F} f(\lambda, b)|c(\lambda)|^{-2} d \lambda d b, \quad(x \in X)
$$

where $c(\lambda)$ is the Harish-Chandra $c$-function, for $f \in C_{c}^{\infty}(X)$. Moreover, $\mathcal{F} f \in \mathcal{H}_{R}\left(\mathfrak{a}_{\mathbb{C}}^{*} \times B\right)^{W}$ if, and only if, $\operatorname{supp} f \subset B_{R}$. We note that $|c(\lambda)|^{-2}$ is bounded by some polynomial for $\lambda \in \mathfrak{a}^{*}$.

Let $f_{1}, f_{2} \in C_{c}^{\infty}(X)$, then Parseval's formula for $\mathcal{F}$ is given by:

$$
\int_{X} f_{1}(x) \overline{f_{2}(x)} d x=\int_{\mathfrak{a}_{+}^{*}} \int_{B} \mathcal{F} f_{1}(\lambda, b) \overline{\mathcal{F} f_{2}(\lambda, b)}|c(\lambda)|^{-2} d \lambda d b .
$$

We conclude that the Fourier transform extends to an isometry of $L^{2}(X)$ onto $L^{2}\left(\mathfrak{a}_{+}^{*} \times B,|c(\lambda)|^{-2} d \lambda d b\right)$. In the following we adopt the convention $L^{2}\left(\mathfrak{a}_{+}^{*} \times B\right):=L^{2}\left(\mathfrak{a}_{+}^{*} \times B,|c(\lambda)|^{-2} d \lambda d b\right)$. 
Let $f \in C^{\infty}(X)$ such that $\Delta^{n} f \in L^{2}(X)$ for all $n \in \mathbb{N} \cup\{0\}$ and let $f_{2} \in C_{c}^{\infty}(X)$. Then self-adjointness of the Laplace-Beltrami operator $\Delta$ :

$$
\int_{X} \Delta^{n} f(x) f_{2}(x) d x=\int_{X} f(x) \Delta^{n} f_{2}(x) d x,
$$

Parseval's formula (5) and density of $C_{c}^{\infty}(X)$ imply, as in the classical case, that

$$
\mathcal{F}\left(\Delta^{n} f\right)(\lambda, b)=(-1)^{n}\left(\|\lambda\|^{2}+\|\rho\|^{2}\right)^{n} \mathcal{F} f(\lambda, b),
$$

a.e., for all $n \in \mathbb{N} \cup\{0\}$.

\section{The inverse Fourier transform.}

We define the inverse Fourier transform $\mathcal{F}^{-1} g$ of a function $g$ on $\mathfrak{a}_{+}^{*} \times B$ via (4):

$$
\mathcal{F}^{-1} g(x):=\int_{\mathfrak{a}_{+}^{*}} \int_{B} e^{(i \lambda+\rho)(A(x, b))} g(\lambda, b)|c(\lambda)|^{-2} d \lambda d b,
$$

for all $x \in X$ for which the integral exists.

We define the support, $\operatorname{supp} g$, of $g \in L^{2}\left(\mathfrak{a}_{+}^{*} \times B\right)$ to be the smallest closed set in $\mathfrak{a}_{+}^{*} \times B$, outside which the function $g$ vanishes almost everywhere, and $R_{g}:=\sup _{(\lambda, b) \in \operatorname{supp} g}\|\lambda\|$ to be the 'radius' of the support of $g$.

Lemma 5.1. Let $g \in L^{2}\left(\mathfrak{a}_{+}^{*} \times B\right)$ such that $\|\lambda\|^{2 n} g(\lambda, b) \in L^{2}\left(\mathfrak{a}_{+}^{*} \times B\right)$ for all $n \in \mathbb{N} \cup\{0\}$. Then

$$
R_{g}=\lim _{n \rightarrow \infty}\left\{\int_{\mathfrak{a}_{+}^{*}} \int_{B}\|\lambda\|^{4 n}|g(\lambda, b)|^{2}|c(\lambda)|^{-2} d \lambda d b\right\}^{1 / 4 n} .
$$

Proof. As for Lemma 2.1.

Let $L_{c}^{2}\left(\mathfrak{a}_{+}^{*} \times B\right)$ denote the subspace of $L^{2}\left(\mathfrak{a}_{+}^{*} \times B\right)$ of functions with bounded support and let $L_{R}^{2}\left(\mathfrak{a}_{+}^{*} \times B\right):=\left\{g \in L_{c}^{2}\left(\mathfrak{a}_{+}^{*} \times B\right) \mid R_{g}=R\right\}$.

Definition 5.2. We define the $L^{2}$-Paley-Wiener space $\mathrm{PW}^{2}(X)$ as the space of all functions $f \in C^{\infty}(X)$ satisfying:

(a) $\Delta^{n} f \in L^{2}(X)$ for all $n \in \mathbb{N} \cup\{0\}$.

(b) $R_{f}^{\Delta}:=\lim _{n \rightarrow \infty}\left\|\left(\Delta+\|\rho\|^{2}\right)^{n} f\right\|_{2}^{1 / 2 n}<\infty$.

Let also $\mathrm{PW}_{R}^{2}(X):=\left\{f \in \mathrm{PW}^{2}(X) \mid R_{f}^{\Delta}=R\right\}$, for $R \geq 0$.

The real $L^{2}$-Paley-Wiener theorem for the inverse Fourier transform can now be formulated as follows:

Theorem 5.3. The inverse Fourier transform $\mathcal{F}^{-1}$ is a bijection of $L_{c}^{2}\left(\mathfrak{a}_{+}^{*} \times\right.$ $B)$ onto $\mathrm{PW}^{2}(X)$, mapping $L_{R}^{2}\left(\mathfrak{a}_{+}^{*} \times B\right)$ onto $\mathrm{PW}_{R}^{2}(X)$. 
Proof. Let $g \in L_{R}^{2}\left(\mathfrak{a}_{+}^{*} \times B\right)$. Then $\mathcal{F}^{-1} g \in C^{\infty}(X)$ by Lebesgue's dominated convergence theorem. Equation (2) gives, for $D \in \mathbb{D}(X)$,

$$
D\left(\mathcal{F}^{-1} g\right)(x)=\int_{\mathfrak{a}_{+}^{*}} \int_{B} \Gamma(D)(i \lambda) e^{(i \lambda+\rho)(A(x, b))} g(\lambda, b)|c(\lambda)|^{-2} d \lambda d b,
$$

which in particular shows that $(\Delta+\|\rho\|)^{n} \mathcal{F}^{-1} g=\mathcal{F}^{-1}\left((-1)^{n}\|\lambda\|^{2 n} g\right) \in$ $L^{2}(X)$ for all $n \in \mathbb{N} \cup\{0\}$. Parseval's formula (5) with

$$
f_{1}=f_{2}=\mathcal{F}^{-1}\left((-1)^{n}\|\lambda\|^{2 n} g\right)
$$

yields:

$$
\begin{aligned}
& \lim _{n \rightarrow \infty}\left\{\int_{X}\left|(\Delta+\|\rho\|)^{n}\left(\mathcal{F}^{-1} g\right)(x)\right|^{2} d x\right\}^{1 / 4 n} \\
& =\lim _{n \rightarrow \infty}\left\{\int_{\mathfrak{a}_{+}^{*}} \int_{B}\|\lambda\|^{4 n}|g(\lambda, b)|^{2}|c(\lambda)|^{-2} d \lambda d b\right\}^{1 / 4 n}=R,
\end{aligned}
$$

whence $\mathcal{F}^{-1} g \in \mathrm{PW}_{R}^{2}(X)$.

Let now $f \in \mathrm{PW}_{R}^{2}(X)$. Then $\mathcal{F}\left((\Delta+\|\rho\|)^{n} f\right)(\lambda, b)=(-1)^{n}\|\lambda\|^{2 n} \mathcal{F} f(\lambda, b)$ $\in L^{2}\left(\mathfrak{a}_{+}^{*} \times B\right)$ for all $n \in \mathbb{N}$ by $(7)$. Another application of Parseval's formula as above with $f_{1}=f_{2}=(\Delta+\|\rho\|)^{n} f$ shows that $R_{\mathcal{F} f}=R_{f}^{\Delta}=R$, and we conclude that $\mathcal{F} f$ has bounded support.

Corollary 5.4. Let $f \in C^{\infty}(X)$ be such that $\Delta^{n} f \in L^{2}(X)$ for all $n \in$ $\mathbb{N} \cup\{0\}$. It then follows that $\lim _{n \rightarrow \infty}\left\|\Delta^{n} f\right\|_{2}^{1 / 2 n}<\infty$ if, and only if, $\lim _{n \rightarrow \infty}\left\|\left(\Delta+\|\rho\|^{2}\right)^{n} f\right\|_{2}^{1 / 2 n}<\infty$. Furthermore, $\lim _{n \rightarrow \infty}\left\|\Delta^{n} f\right\|_{2}^{1 / 2 n}=\left(R^{2}+\right.$ $\left.\|\rho\|^{2}\right)^{1 / 2}$, for $f \in \mathrm{PW}_{R}^{2}(X)$ with $R>0$.

Proof. Let $f \in \mathrm{PW}_{R}^{2}(X)$, with $R>0$, then $\mathcal{F} f \in L_{R}^{2}\left(\mathfrak{a}_{+}^{*} \times B\right)$. Parseval's formula and an easy adaption of the proof of Lemma 2.1 shows that

$$
\begin{aligned}
& \lim _{n \rightarrow \infty}\left\|\Delta^{n} f\right\|_{2}^{1 / 2 n} \\
& =\lim _{n \rightarrow \infty}\left\{\int_{\mathfrak{a}_{+}^{*}} \int_{B}\left(\|\lambda\|^{2}+\|\rho\|^{2}\right)^{2 n}|\mathcal{F} f(\lambda, b)|^{2}|c(\lambda)|^{-2} d \lambda d b\right\}^{1 / 4 n} \\
& =\left(R^{2}+\|\rho\|^{2}\right)^{1 / 2} .
\end{aligned}
$$


Assume that $\lim _{n \rightarrow \infty}\left\|\Delta^{n} f\right\|_{2}^{1 / 2 n}<\infty$. Then $\mathcal{F}\left(\Delta^{n} f\right)(\lambda, b)=(-1)^{n}\left(\|\lambda\|^{2}\right.$ $\left.+\|\rho\|^{2}\right)^{n} \mathcal{F} f(\lambda, b) \in L^{2}\left(\mathfrak{a}_{+}^{*} \times B\right)$, for all $n \in \mathbb{N}$, and

$$
\begin{aligned}
& \lim _{n \rightarrow \infty}\left\{\int_{\mathfrak{a}_{+}^{*}} \int_{B}\|\lambda\|^{4 n}|\mathcal{F} f(\lambda, b)|^{2}|c(\lambda)|^{-2} d \lambda d b\right\}^{1 / 4 n} \\
& \leq \lim _{n \rightarrow \infty}\left\{\int_{\mathfrak{a}_{+}^{*}} \int_{B}\left(\|\lambda\|^{2}+\|\rho\|^{2}\right)^{2 n}|\mathcal{F} f(\lambda, b)|^{2}|c(\lambda)|^{-2} d \lambda d b\right\}^{1 / 4 n} \\
& =\lim _{n \rightarrow \infty}\left\|\Delta^{n} f\right\|_{2}^{1 / 2 n}<\infty,
\end{aligned}
$$

that is, $\mathcal{F} f$ has bounded support.

Remark 5.5. Assume that $f \in \mathcal{S}^{p}(X)$, with $0<p<2$, then $\mathcal{F} f$ extends to an analytic function on a small tube domain around $\mathfrak{a}^{*} \times B$ in $\mathfrak{a}_{\mathbb{C}}^{*} \times B$. Hence $\mathcal{F} f$ cannot have compact support on $\mathfrak{a}^{*} \times B$ and we conclude that $\mathcal{S}^{p}(X) \cap \mathrm{PW}^{2}(X)=\{0\}$ for any $0<p<2$.

Definition 5.6. We define the Paley-Wiener space $\mathrm{PW}(X)$ as the space of all functions $f \in C^{\infty}(X)$ satisfying:

(a) $(1+|x|)^{m} \Delta^{n} f \in L^{2}(X)$ for all $m, n \in \mathbb{N} \cup\{0\}$.

(b) $R_{f}^{\Delta}=\lim _{n \rightarrow \infty}\left\|\left(\Delta+\|\rho\|^{2}\right)^{n} f\right\|_{2}^{1 / 2 n}<\infty$.

Let also $\mathrm{PW}_{R}(X):=\left\{f \in \mathrm{PW}(X) \mid R_{f}^{\Delta}=R\right\}$, for $R \geq 0$.

Here $|x|:=|g|$, for $x=g K \in X$. Again, the only difference between the Paley-Wiener spaces $\mathrm{PW}(X)$ and $\mathrm{PW}^{2}(X)$ is the polynomial decay condition (a), ensuring that $\mathcal{F} f \in C^{\infty}\left(\mathfrak{a}^{*} \times B\right)^{W}$ (see below).

The space $C_{c}^{\infty}\left(\mathfrak{a}^{*} \times B\right)^{W}$ is defined as the subspace of functions $\psi \in$ $C_{c}^{\infty}\left(\mathfrak{a}^{*} \times B\right)$ satisfying the symmetry condition (3) for all $w \in W$ and all $\lambda \in \mathfrak{a}^{*}, x \in X$. Let finally $C_{R}^{\infty}\left(\mathfrak{a}^{*} \times B\right):=\left\{F \in C_{c}^{\infty}\left(\mathfrak{a}^{*} \times B\right) \mid R_{g}=R\right\}$.

The real Paley-Wiener theorem for the inverse Fourier transform then is:

Theorem 5.7. The inverse Fourier transform $\mathcal{F}^{-1}$ is a bijection of $C_{c}^{\infty}\left(\mathfrak{a}^{*} \times\right.$ $B)^{W}$ onto $\mathrm{PW}(X)$, mapping $C_{R}^{\infty}\left(\mathfrak{a}^{*} \times B\right)^{W}$ onto $\mathrm{PW}_{R}(X)$.

Proof. Let $g \in C_{R}^{\infty}\left(\mathfrak{a}^{*} \times B\right)^{W}$, then $g \in L_{R}^{2}\left(\mathfrak{a}_{+}^{*} \times B\right)$ and thus $\mathcal{F}^{-1} g \in$ $\mathrm{PW}_{R}^{2}(X)$ by Theorem 5.3. We furthermore see that $\mathcal{F}^{-1} g \in \mathcal{S}^{2}(X)$ by [4, Theorem 4.1.1], whence $\mathcal{F}^{-1} g$ satisfies the polynomial decay condition (a).

Let now $f \in \mathrm{PW}_{R}(X)$. The basic estimate $\|A(g)\| \leq C|g|$, for all $g \in G$, gives us a polynomial estimate (in $x$ ) of the derivatives (with respect to $\lambda$ ) of the plane wave eigenfunctions $e_{\lambda, b}(x)$. It is also well-known that $(1+|x|)^{-r} \varphi_{0} \in L^{2}(X)$ for some large $r \in \mathbb{N}$. All this, the polynomial decay condition (a), the Cauchy-Schwartz theorem and Lebesgue's dominated convergence theorem imply that $\mathcal{F} f \in C^{\infty}\left(\mathfrak{a}^{*} \times B\right)^{W}$. Furthermore $\mathcal{F} f$ has the desired compact support by Theorem 5.3. 


\section{The inverse spherical transform.}

In this section, we specialise our results to bi- $K$-invariant functions, that is, we consider the (inverse) spherical transform. We refer to [1], [5] and [6] for background concerning Paley-Wiener theorems for the spherical transform. Let $C^{\infty}(K \backslash G / K) \subset C^{\infty}(G)$ denote the subspace of bi- $K$-invariant differentiable functions on $G$. We will use similar notation for the $L^{2}$, Paley-Wiener and Schwartz spaces of $K$-invariant differentiable functions.

Let $f \in C_{c}^{\infty}(K \backslash G / K)$. The spherical transform $\mathcal{H} f$ of $f$ is defined as:

$$
\mathcal{H} f(\lambda):=\int_{G} f(x) \varphi_{-\lambda}(x) d x,
$$

for $\lambda \in \mathfrak{a}_{\mathbb{C}}^{*}$. We note that $\mathcal{F} f(\lambda, b)=\mathcal{H} f(\lambda)$ for all $\lambda \in \mathfrak{a}_{\mathbb{C}}^{*}$ and all $b \in B$. This follows from left- $K$-invariance of $f$, the identity $A(k \cdot x, b)=A\left(x, k^{-1} \cdot b\right)$ and integrating over $K$.

The spherical transform is an isomorphism of $\mathcal{S}^{2}(K \backslash G / K)$ onto $\mathcal{S}\left(\mathfrak{a}^{*}\right)^{W}$, the Weyl group invariant Schwartz functions on $\mathfrak{a}^{*}$. The inversion formula is given by:

$$
f(x)=\frac{1}{|W|} \int_{\mathfrak{a}^{*}} \mathcal{H} f(\lambda) \varphi_{\lambda}(x)|c(\lambda)|^{-2} d \lambda, \quad(x \in G)
$$

for $f \in \mathcal{S}^{2}(K \backslash G / K)$. We use (8) to define the inverse spherical transform $\mathcal{H}^{-1} g$ for a general function $g$ on $\mathfrak{a}^{*}$ :

$$
\mathcal{H}^{-1} g(x):=\frac{1}{|W|} \int_{\mathfrak{a}^{*}} g(\lambda) \varphi_{\lambda}(x)|c(\lambda)|^{-2} d \lambda .
$$

Let $f \in C^{\infty}(K \backslash G / K)$ be such that $\Delta^{n} f \in L^{2}(K \backslash G / K)$ for all $n \in$ $\mathbb{N} \cup\{0\}$. Then $\mathcal{H}\left(\left(\Delta+\|\rho\|^{2}\right)^{n} f\right)(\lambda)=(-1)^{n}\|\lambda\|^{2 n} \mathcal{H} f(\lambda)$ a.e., and Parseval's formula for $\mathcal{H}$ gives:

$$
\int_{G}\left|\left(\Delta+\|\rho\|^{2}\right)^{n} f(x)\right|^{2} d x=\frac{1}{|W|} \int_{\mathfrak{a}^{*}}\|\lambda\|^{4 n}|\mathcal{H} f(\lambda)|^{2}|c(\lambda)|^{-2} d \lambda,
$$

for all $n \in \mathbb{N} \cup\{0\}$. It also follows that the spherical transform extends to an isometry from $L^{2}(K \backslash G / K)$ onto $L^{2}\left(\mathfrak{a}^{*}, \frac{1}{|W|}|c(\lambda)|^{-2} d \lambda\right)^{W}$, where superscript $W$ denotes Weyl group invariance.

Let $L_{c}^{2}\left(\mathfrak{a}^{*}\right)^{W}$ denote the Weyl group invariant $L^{2}$-functions on $\mathfrak{a}^{*}$ with compact support and let subscript $R$ denote the radius of the support. The real versions of the Paley-Wiener theorems for the inverse spherical transform then becomes:

Theorem 6.1. The inverse spherical transform $\mathcal{H}^{-1}$ is a bijection of $L_{c}^{2}\left(\mathfrak{a}^{*}\right)^{W}$ onto $\mathrm{PW}^{2}(K \backslash G / K)$, mapping $L_{R}^{2}\left(\mathfrak{a}^{*}\right)^{W}$ onto $\mathrm{PW}_{R}^{2}(K \backslash G / K)$.

Theorem 6.2. The inverse spherical transform $\mathcal{H}^{-1}$ is a bijection of $C_{c}^{\infty}\left(\mathfrak{a}^{*}\right)^{W}$ onto $\mathrm{PW}(K \backslash G / K)$, mapping $C_{R}^{\infty}\left(\mathfrak{a}^{*}\right)^{W}$ onto $\mathrm{PW}_{R}(K \backslash G / K)$. 
Proof. The above theorems are special cases of Theorem 5.3 and Theorem 5.7. We note, however, that we can prove them independently using Parseval's formula and intertwining properties of $\mathcal{H}$.

Remark 6.3. Let $f \in \mathrm{PW}(K \backslash G / K)$ and consider $f$ as a function on $\mathfrak{a}$ by the application $H \mapsto f(\exp (H))$. Then $f$ does not extend to an entire function on $\mathfrak{a}_{\mathbb{C}}$, due to the poles of the spherical function $\varphi_{\lambda}(\exp (H))$. There is, however, a description of the Paley-Wiener space PW $(K \backslash G / K)$ as functions having an explicit meromorphic extension and satisfying some exponential growth conditions for the rank 1 and the complex cases, see [8] for details.

\section{References}

[1] J.-Ph. Anker, The spherical Fourier transform of rapidly decreasing functions. A simple proof of a characterization due to Harish-Chandra, Helgason, Trombi, and Varadarajan, J. Funct. Anal., 96 (1991), 331-349, MR 92d:22008, Zbl 0732.43006.

[2] H.H. Bang, A property of infinitely differentiable functions, Proc. Amer. Math. Soc., 108 (1990), 73-76, Zbl 0707.26015.

[3] _ Functions with bounded spectrum, Trans. Amer. Math. Soc., 347 (1995), 1067-1080, MR 95e:42008, Zbl 0828.42009.

[4] M. Eguchi, Asymptotic expansions of Eisenstein integrals and Fourier transform on symmetric spaces, J. Funct. Anal., 34 (1979), 167-216, MR 81e:43022, Zbl 0433.43012.

[5] R. Gangolli and V.S. Varadarajan, Harmonic Analysis of Spherical Functions on Real Reductive Groups, Springer-Verlag, Berlin, 1988, MR 89m:22015, Zbl 0675.43004.

[6] S. Helgason, Groups and Geometric Analysis, Academic Press, Orlando, 1984, MR 86c:22017, Zbl 0543.58001.

[7] _ Geometric Analysis on Symmetric Spaces, Mathematical Surveys and Monographs, 39, American Mathematical Society, Providence, Rhode Island, 1994, MR 96h:43009, Zbl 0809.53057.

[8] A. Pasquale, A Paley-Wiener theorem for the inverse spherical transform, Pacific J. Math., 193 (2000), 143-176, MR 2001c:22011, Zbl 1014.22010.

[9] W. Rudin, Functional Analysis, McGraw-Hill, New York, 1991, MR 92k:46001, Zbl 0867.46001.

[10] V.K. Tuan, Paley-Wiener-type theorems, Fract. Calc. Appl. Anal., 2 (1999), 135-143.

[11] V.K. Tuan and A.I. Zayed, Paley-Wiener-type theorems for a class of integral transforms, J. Math. Anal. Appl., 266 (2002), 200-226, MR 2002k:44005, Zbl 0998.44001.

Received October 14, 2002 and revised February 11, 2003. The author is supported by a research grant from the Australian Research Council.

SChool of Mathematics

University of New South WaLes

SYDNEY NSW 2052

Australia

E-mail address: byrial@maths.unsw.edu.au 